\title{
SPALL DAMAGE IN ALUMINUM ALLOY
}

\author{
JIAN ZHENG \\ Laboratory for Non-linear Mechanics of Continuous Media, Institute of Mechinics, \\ Chinese Academy of Science, Beijing 100080, People's Republic of China \\ and \\ ZE-PING WANG \\ Department of Mechanical and Production Engineering, National University of Singapore, \\ 10 Kent Ridge Crescent, Singapore 0511
}

(Received 15 December 1993 ; in revised form 8 July 1994)

\begin{abstract}
A void growth relation for ductile porous materials under intense dynamic general loading conditions is presented. The mathematical model includes the influence of inertial effects, material rate sensitivity, as well as the contribution of void surface energy and material workhardening. Numerical analysis shows that inertia appears to resist the growth of voids. The inertial effects increase quickly with the loading rates. The theoretical analysis suggests that the inertial effects cannot be neglected at high loading rates. Plate-impact tests of aluminum alloy are performed with light gas gun. The processes of dynamic damage in aluminum alloy are successfully simulated with a finite-difference dynamic code in which the theoretical model presented in this paper is incorporated.
\end{abstract}

\section{INTRODUCTION}

Dynamic ductile fracture with different loading conditions, such as high speed impact, explosive loading, dynamic tension of smooth or notched bar specimens, is a consequence of the nucleation, growth and coalescence of voids in a triaxial stress field. In comparison to ductile damage under static loading, dynamic ductile damage is much more complex. The inertial effects, rate-dependence and thermal influence from rapid plastic deformation are the characteristics under intense dynamic loading. The literature on this subject is extensive. By investigating the behavior of dynamic damage and fracture in solids in detail, Curran and co-workers (Barber et al., 1972; Seaman et al., 1976; Curran et al., 1977, 1987) established computational models called NAG (nucleation and growth) models for ductile and brittle fracture. In their models, two internal state variables $N$ (the number of microvoids or microcracks per unit volume) and $R$ (the average size of a microvoid or microcrack) are introduced to describe the processes of dynamic damage and fracture in solids. The NAG models have sufficient generality to include the statistical distribution of one or more variables such as porosity, void density, etc., but require numerous phenomenological constants that are difficult to obtain. Carroll and Holt (1972) developed static and dynamic pore-collapse relations for ductile porous materials. The material was assumed to be rate insensitive and ideally plastic. They suggested that the effect of elastic compressibility in the matrix material is small, and can go immediately to the case of fully plastic deformation around the void. Johnson (1981) applied Carroll and Holt's approach to void growth in a viscoplastic medium. Cochran and Banner (1977) studied spallation in uranium using a simple theoretical model. Rajendran et al. (1989) proposed a new dynamic failure model to describe void nucleation, growth and coalescence in ductile metals. Nash (Nash and Cullis 1984; Nash, 1985) directly used Rice's static model to model ductile fracture in triaxial states of stress. Cortes (1992) adopted Carroll and Holt's assumption to investigate the growth of a microvoid under intense dynamic loading. Review articles (Meyers and Aimone 1983; Curran et al., 1987; Grady, 1988) on dynamic ductile fracture explain in some detail the most relevant results of experimental and theoretical studies. 


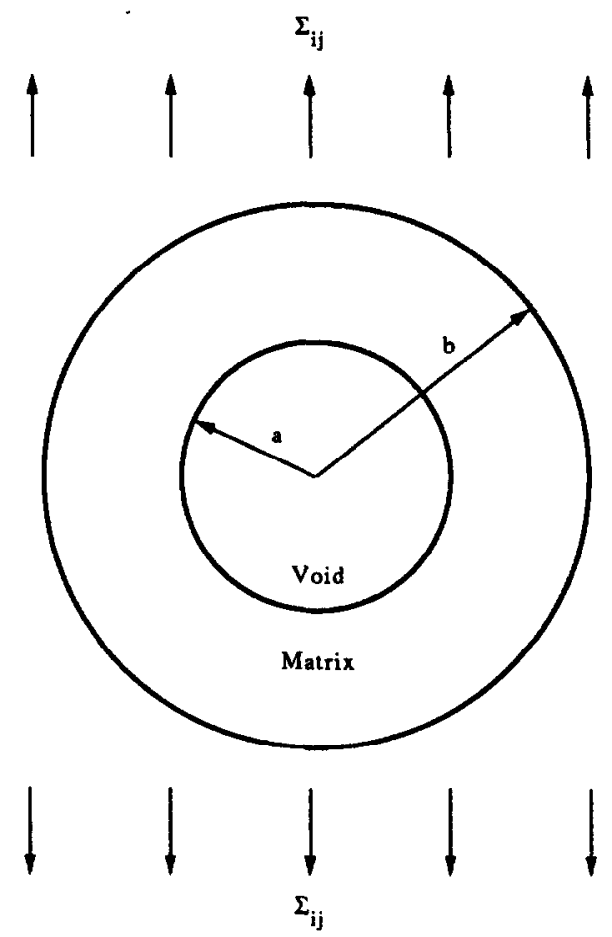

Fig. 1. A spherical element of material of radius $b$ containing a single void of radius $a$ is subjected to an applied external stress $\Sigma_{i j}$.

The inertial effects are the major feature of intense dynamic loading. Our theoretical analysis shows that their influence on the growth of dynamic ductile voids is significant. Since the problem is quite complicated, most investigators neglected them.

The influence of deviatoric stresses is also important for most of the processes of dynamic ductile fracture. Although in the past several authors have studied the void growth problem under triaxiality conditions (Rice and Tracey, 1969; Gurson, 1977; Duva and Hutchinson, 1984; Cocks, 1989), they have limited their analysis to static loading, ignoring the influence of inertial effects. In this paper, we deal with dynamic growth of voids in ductile materials under extremely high rates of general loading. A void growth relation, in which the inertial effects, rate-dependence, the contribution of the void surface energy are considered, is presented by means of the energy principles.

To simplify theoretical analysis, we assume that the matrix material is incompressible during the void growth. We also assume that the void remains spherical all the time. These assumptions lead to great simplification of the theoretical analysis, so that we can obtain the exact relation for void growth.

\section{VOID GROWTH RELATION}

We assume that the porous material consists of a suspension of pores in a matrix of homogeneous isotropic ductile solid materials which is subjected to an external stress $\Sigma_{i j}$, and that the porous material is statistically homogeneous and isotropic so that it can be effectively modeled by a homogeneous isotropic solid material. With these assumptions, we can study the void growth by considering a hole sphere with inner radius $a$ and outer radius $b$ (see Fig. 1). Distention $\alpha$ is defined as

$$
\alpha=\frac{b^{3}}{b^{3}-a^{3}}
$$

We investigate the response of this hole sphere to time-dependent external stress and zero internal pressure, and try to obtain the relation between the applied stress $\Sigma_{i j}$ and 
distention $\alpha(t)$. We expect that the relation will adequately describe the void growth for the effective homogeneous material.

Taking the matrix material and void as a system, work done by the applied external stress $\Sigma_{i j}$ is equal to the change of the system energy, namely

$$
W=\Delta E_{\mathrm{K}}+\Delta E_{\mathrm{s}}+\Delta E_{\mathrm{i}}
$$

where $\Delta E_{\mathrm{K}}, \Delta E_{\mathrm{S}}$ and $\Delta E_{\mathrm{i}}$ denote the changes of the kinetic energy $E_{\mathrm{K}}$, the void surface energy $E_{\mathrm{S}}$, and the internal energy $E_{\mathrm{i}}$ in the system, respectively. $W$ denotes the work done by the applied external stress $\Sigma_{i j}$. Since the contribution of the initial elastic and elasticplastic phases of the process for the void growth is small (Carroll and Holt, 1972), we directly begin to consider fully plastic deformation in the solid around the void. From the assumption of incompressibility in the matrix material surrounding the void, the following expressions can be derived (Johnson, 1981):

$$
\begin{gathered}
r^{3}=r_{0}^{3}-B(t) \\
B(t)=a_{0}^{3} \frac{\alpha_{0}-\alpha}{\alpha_{0}-1} \\
\frac{B(t)}{a^{3}}=\frac{\alpha_{0}-\alpha}{\alpha-1} \\
\frac{B(t)}{b^{3}}=\frac{\alpha_{0}-\alpha}{\alpha},
\end{gathered}
$$

where $r$ is Eulerian radial position of a Lagrangian point that travels with the material. The initial radius of that point is $r_{0} . B(t)$ is a function related to the rate of void growth. $a_{0}$ is the initial radius of the void. Let us consider $\Delta E_{\mathrm{K}}, \Delta E_{\mathrm{S}}, \Delta E_{\mathrm{i}}$ and $W$, respectively. $\Delta E_{\mathrm{K}}$ is given by

$$
\Delta E_{\mathrm{K}}=E_{\mathrm{K}}(\alpha)-E_{\mathrm{K}}\left(\alpha_{0}\right)
$$

with

$$
E_{\mathrm{K}}(\alpha)=\int_{a}^{b} \frac{1}{2} \dot{r}^{2} \rho_{\mathrm{s}} 4 \pi r^{2} \mathrm{~d} r
$$

where () denotes the differential with respect to time $t . \rho_{\mathrm{S}}$ is the density of the matrix material. With the aim of eqn (1) and eqns (3)-(6), eqn (8) becomes

$$
E_{\mathbf{K}}(\alpha)=\left[\frac{4 \pi a_{0}^{3}}{9\left(\alpha_{0}-1\right)}\right] \frac{a_{0}^{2} \rho_{\mathrm{s}}}{2\left(\alpha_{0}-1\right)}\left(\frac{\alpha_{0}-1}{\alpha-1}\right)^{1 / 3}\left[1-\left(\frac{\alpha-1}{\alpha}\right)^{1 / 3}\right] \dot{\alpha}^{2} .
$$

In the same way, we can also obtain the expression of $\Delta E_{\mathrm{S}}$ :

$$
\Delta E_{\mathrm{S}}=E_{\mathrm{S}}(\alpha)-E_{\mathrm{S}}\left(\alpha_{0}\right)
$$

with

$$
E_{\mathrm{S}}(\alpha)=\left[\frac{4 \pi a_{0}^{3}}{9\left(\alpha_{0}-1\right)}\right] \frac{9\left(\alpha_{0}-1\right)^{1 / 3} \gamma}{a_{0}}(\alpha-1)^{2 / 3},
$$

where $\gamma$ is the surface energy expended per unit area during the hole expansion.

The matrix material is assumed to be linear work-hardening, and viscoplastic. Constitutive relation is supposed to be (Perzyna, 1986) 


$$
\sigma_{\mathrm{eqv}}=Y_{0}+H \varepsilon_{\mathrm{eqv}}^{\mathrm{P}}+\eta \dot{\varepsilon}_{\mathrm{eqv}}^{\mathrm{p}}
$$

where $\sigma_{\text {eqv }}, \varepsilon_{\text {eqv }}^{\mathrm{P}}$ and $\dot{\varepsilon}_{\text {eqv }}^{\mathrm{P}}$ denote the effective stress, the effective plastic strain and the effective plastic strain rate in the matrix material, respectively. $Y_{0}$ is the yield stress of the matrix material, $H$ is a linear work-hardening coefficient, and $\eta$ is the material viscosity. Since we assume a plastic deformation process with spherical symmetry, the effective plastic strain $\varepsilon_{\mathrm{eqv}}^{\mathrm{P}}$ is given by Johnson and Mellor (1973) :

$$
\varepsilon_{\mathrm{eqv}}^{\mathrm{P}}=2 \ln \frac{r}{r_{0}}
$$

The change of internal energy $\Delta E_{\mathrm{i}}$ in the system is

$$
\Delta E_{\mathrm{i}}=\frac{1}{\rho} \int_{a}^{b}\left[\int_{0}^{\delta_{\text {eqv }}^{\mathrm{P}}} \sigma_{\text {eqv }}\left(\varepsilon^{\mathrm{P}}\right) \mathrm{d} \varepsilon^{\mathrm{P}}\right] 4 \pi \rho r^{2} \mathrm{~d} r
$$

By means of eqns (3)-(6) and eqns (12)-(13), we finally have

$$
\Delta E_{\mathrm{i}}=\left[\frac{4 \pi a_{0}^{3}}{9\left(\alpha_{0}-1\right)}\right]\left[F_{3}(\alpha)+F_{4}(\alpha)+F_{5}(\alpha) \dot{\alpha}\right]
$$

where

$$
\begin{gathered}
F_{3}(\alpha)=2 Y_{0}\left(\ln \frac{\alpha-1}{\alpha_{0}-1}+\alpha \ln \frac{\alpha}{\alpha-1}-\alpha_{0} \ln \frac{\alpha_{0}}{\alpha_{0}-1}\right) \\
F_{4}(\alpha)=\frac{4}{3} H\left[\left(\alpha-\alpha_{0}\right) F(\alpha)+\frac{\alpha_{0}}{2}\left(\ln \frac{\alpha_{0}}{\alpha}\right)^{2}-\frac{\alpha_{0}-1}{2}\left(\ln \frac{\alpha_{0}-1}{\alpha-1}\right)^{2}\right] \\
F(\alpha)=\int_{h_{0}}^{h_{1}} \frac{\ln (h+1)}{h} \mathrm{~d} h,
\end{gathered}
$$

with

$$
h_{0}=\frac{\alpha_{0}-\alpha}{\alpha-1}, \quad h_{1}=\frac{\alpha_{0}-\alpha}{\alpha}, \quad F_{5}(\alpha)=\frac{2}{3} \eta\left(\ln \frac{\alpha-1}{\alpha}+\ln \frac{\alpha_{0}}{\alpha_{0}-1}\right)
$$

Functions $F_{3}(\alpha), F_{4}(\alpha)$ and $F_{5}(\alpha)$ denote the influence of the yield stress of the matrix material, the linear work-hardening and the material viscosity on the increment of internal energy, respectively.

The work $W$ done by external stress is as follows:

$$
W=\frac{4}{3} \pi \frac{a_{0}^{3}}{\alpha_{0}-1} \int_{\alpha_{0}}^{\alpha}\left\lfloor\frac{2}{3} \Sigma_{\text {eqv }}\left(\alpha^{\prime}\right)-P\left(\alpha^{\prime}\right)\right] \mathrm{d} \alpha^{\prime}
$$

Here we assume $\Sigma_{\text {eqv }}$ and $P$ to be the functions of distention $\alpha$, that is, $\Sigma_{\text {eqv }}=\Sigma_{\text {eqv }}(\alpha)$ and $P=P(\alpha)$. Substitution of expressions of $\Delta E_{\mathrm{K}}, \Delta E_{\mathrm{s}}, \Delta E_{\mathrm{i}}$ and $W$ into eqn (2) gives

$$
F_{1}(\alpha) \dot{\alpha}^{2}+F_{5}(\alpha) \dot{\alpha}+F_{7}(\alpha)=0
$$

where 


$$
\begin{gathered}
F_{1}(\alpha)=\frac{\rho a_{0}^{2}}{2\left(\alpha_{0}-1\right)}\left(\frac{\alpha_{0}-1}{\alpha-1}\right)^{1 / 3}\left[1-\left(\frac{\alpha-1}{\alpha}\right)^{1 / 3}\right] \\
F_{7}(\alpha)=F_{2}(\alpha)+F_{3}(\alpha)+F_{4}(\alpha)-3 \int_{\alpha_{0}}^{\alpha}\left[\frac{2}{3} \Sigma_{\text {eqv }}\left(\alpha^{\prime}\right)-P\left(\alpha^{\prime}\right)\right] \mathrm{d} \alpha^{\prime}-F_{1}\left(\alpha_{0}\right) \dot{\alpha}_{0}^{2}-F_{2}\left(\alpha_{0}\right) \\
F_{2}(\alpha)=\frac{9\left(\alpha_{0}-1\right)^{1 / 3} \gamma}{a_{0}}(\alpha-1)^{2 / 3} .
\end{gathered}
$$

Equation (21) is the relationship from which we obtain the rate-dependent response of the void growth under dynamic loading. $F_{2}(\alpha)$ represents the influence of change of the void surface energy on the void growth. The terms in eqn (21) have a clear physical significance. The first term $F_{1}(\alpha) \dot{\alpha}^{2}-F_{1}\left(\alpha_{0}\right) \dot{\alpha}_{0}^{2}$ on the left of eqn (21) represents inertial resistance to the void growth. The second term $F_{5}(\alpha) \dot{\alpha}$ denotes the influence of the material viscosity, which describes the effect of the rate sensitivity and is one of the major features differing from the quasi-static growth of voids. Other researchers' studies, such as Curran et al. (1987), Johnson (1981) and Cortes (1992), have resulted in the same fact. The third term $F_{7}(\alpha)$ is the total effects of the applied external stress, the change of the void surface energy, work-hardening and the yield stress in the solid surrounding the void on the void growth. These effects also can be isolated and studied in great depth. Dynamic ductile fracture is a consequence of the nucleation, growth and coalescence of voids in a triaxial stress field. Besides the mean stress, the deviatoric stress, no doubt, may affect the void growth. The influence of the deviatoric stress (or the deviatoric strain) on the void growth is considered in Rice and Tracey's model (1969) and Gurson's model (1977). A modified Gurson's model was successfully applied to model the cup-cone fracture in a round tensile bar (Tvergaard and Needleman, 1984). But the effect of the deviatoric stress was not included in Carroll and Holt's (1972) as well as Seaman et al.'s (1976) models of dynamic ductile fracture. From eqn (21), the void growth rates $\dot{\alpha}$ can be given by

$$
\dot{\alpha}=\frac{1}{2 F_{1}(\alpha)}\left\{-F_{5}(\alpha)+\sqrt{\left[F_{5}(\alpha)\right]^{2}-4 F_{1}(\alpha) F_{7}(\alpha)}\right\}
$$

Since $\dot{\alpha}$ is a real variable, the following condition must be satisfied for the condition of the void growth:

$$
\left[F_{5}(\alpha)\right]^{2}-4 F_{1}(\alpha) F_{7}(\alpha) \geqslant 0
$$

If the inertial effects are neglected, from eqn $(21), \dot{\alpha}$ is reduced to

$$
\dot{\alpha}=-\left[F_{7}(\alpha)+F_{1}\left(\alpha_{0}\right) \dot{\alpha}_{0}^{2}\right] / F_{5}(\alpha) \text {. }
$$

\section{THRESHOLD STRESSES FOR DYNAMIC GROWTH OF VOIDS}

Define a quantity $\Sigma$ as

$$
\Sigma=\frac{2}{3} \Sigma_{\text {eqv }}-P
$$

Obviously, $\Sigma$ represents the total external stress acting on the spherical element. It shows that either the mean stress $-P$ or the effective stress $\Sigma_{\text {eqv }}$ has a contribution to the void growth. We consider the condition of the void growth, namely, $\dot{\alpha} \geqslant 0$. From eqn (25), the following inequality must be satisfied : 


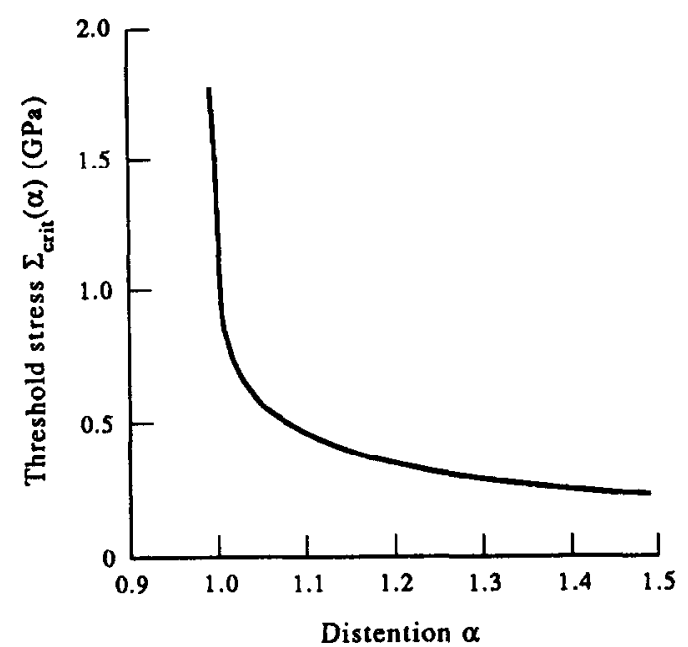

Fig. 2. The threshold stress $\Sigma_{\text {crit }}(\alpha)$ for the void growth decreases along with increase of distention $\alpha$.

$$
\Sigma(\alpha) \geqslant \frac{1}{3}\left[\frac{\mathrm{d} F_{2}(\alpha)}{\mathrm{d} \alpha}+\frac{\mathrm{d} F_{3}(\alpha)}{\mathrm{d} \alpha}+\frac{\mathrm{d} F_{4}(\alpha)}{\mathrm{d} \alpha}\right]
$$

With the help of eqns (16)-(17) and (24), inequality (29) becomes

$$
\Sigma(\alpha) \geqslant \frac{1}{3}\left[\frac{6 \gamma}{a_{0}}\left(\frac{\alpha_{0}-1}{\alpha-1}\right)^{1 / 3}+\frac{4 H}{3} F(\alpha)-\frac{8 H}{3} \frac{\alpha_{0}-1}{\alpha-1} \ln \frac{\alpha-1}{\alpha_{0}-1}+2 Y_{0} \ln \frac{\alpha}{\alpha-1}\right] .
$$

Let

$$
\Sigma_{\text {crit }}(\alpha)=\frac{1}{3}\left[\frac{6 \gamma}{a_{0}}\left(\frac{\alpha_{0}-1}{\alpha-1}\right)^{1 / 3}+\frac{4 H}{3} F(\alpha)-\frac{8 H}{3} \frac{\alpha_{0}-1}{\alpha-1} \ln \frac{\alpha-1}{\alpha_{0}-1}+2 Y_{0} \ln \frac{\alpha}{\alpha-1}\right]
$$

where $\Sigma_{\text {crit }}(\alpha)$ is the threshold stress for dynamic growth of voids in general dynamic loading conditions. The critical conditions that the applied external stress must satisfy for the void growth is that

$$
\Sigma(\alpha) \geqslant \Sigma_{\text {crit }}(\alpha)
$$

If the contributions of the change of the void surface energy and material workhardening, as well as the action of the external deviatoric stress are neglected, eqn (31) is reduced to

$$
P_{\text {crit }}(\alpha)=-\frac{2}{3} Y_{0} \ln \frac{\alpha}{\alpha-1}
$$

This is the result obtained by Carroll and Holt (1972).

\section{NUMERICAL ANALYSIS OF THE MODEL}

In this section, copper-like material is selected to be the material for the numerical analysis with the density $\rho=8.92\left(\mathrm{~g} \mathrm{~cm}^{-3}\right)$, the yield stress $Y_{0}=0.26(\mathrm{GPa})$, the linear work-hardening coefficient $H=0.25(\mathrm{GPa})$, viscosity $\eta=0.1$ (GPa $\mu \mathrm{s})$, and the surface energy expended per unit area $\gamma=9 \times 10^{-4}\left(\mathrm{GPa} \mathrm{cm}^{-2}\right)$.

The relation of the threshold stress $\Sigma_{\text {crit }}(\alpha)$ and distention $\alpha$ for the void growth in terms of eqn (31) is depicted in Fig. 2. It shows that the threshold stress $\Sigma_{\text {crit }}(\alpha)$ decreases quickly as distention $\alpha$ increases. The maximum value of the threshold stress $\Sigma_{\text {crit }}(\alpha)$ is 

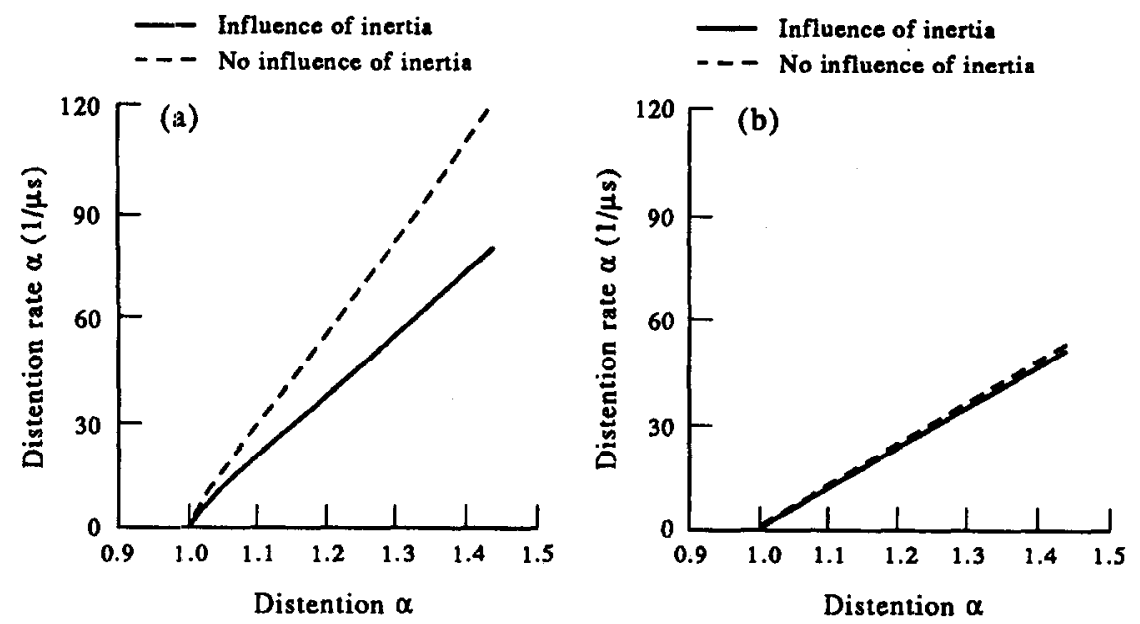

Fig. 3. Influence of inertia on the rate of increase of distention $\alpha$ under the different loading rates. (a) $G=0.3$. (b) $G=0.01$.

about $1.76(\mathrm{GPa})$ which depends on the initial radius of the void $a_{0}$ and the initial distention $\alpha_{0}$. Our experimental observation (Wang, 1993) shows that the initial radius of the void $a_{0}$ is in the range $1-10(\mu \mathrm{m}), a_{0}$ and $\alpha_{0}$ are given by $0.0005(\mathrm{~cm})$ and 1.0003 . The results of spall experiments in copper (Grady, 1988) show that the spall strength of copper is in the range 1.0-2.5 (GPa). Theoretical calculation in this paper is consistent with experimental measurements. The threshold stress of dynamic fracture of voids is larger than that of quasi-static fracture. This may be due to the inertia or the kinetics associated with the micromechanisms controlling the damage process.

To investigate the effects of inertia on the behavior of the void growth under the different loading rate, we numerically analyze eqns (25) and (27). In order to simplify the analysis, the material is assumed to be subjected to a linearly increasing external stress:

$$
\Sigma(\alpha)=\Sigma_{0}+G\left(\alpha-\alpha_{0}\right)
$$

where $\Sigma_{0}=\Sigma_{\text {crit }}\left(\alpha_{0}\right)$ and $G$ is a constant. Figure 3 indicates that the influence of inertial effects becomes larger with the increase of loading rates. The computational results of numerical analysis in Fig. 3 suggest that the influence of inertial effects play, which appears to resist the void growth, an important role in void growth under high rate loading conditions. On the other hand, the numerical analysis in Fig. 3(b) also shows the fact that, if the rate of applied external stress becomes much lower, the inertial effects can be ignored. This result implies that the inertial effects are a mechanical phenomenon which appears significantly in the condition of intense dynamic loading.

\section{APPLICATION OF THE MODEL}

\subsection{Plate-impact tests}

As an application of the foregoing theory, two spall experiments of aluminum alloy are simulated. Specimens were machined into circular plates $70 \mathrm{~mm}$ in diameter. The experiments were performed with a $101 \mathrm{~mm}$ bore single-stage light gas gun. The schematic arrangement for the experiment is shown in Fig. 4. The impact velocities and size of flyers and targets are listed in Table 1 . The specimens were softly recovered with a specially designed catcher to prevent any secondary damage. Measured stress-time histories recorded with a manganin gauge in two spalled specimens of aluminum alloy are shown in Fig. 5. It shows clearly the loading plateau and the signals of spallation. Peak stresses taken from the manganin gauge reading are listed in Table 1. 


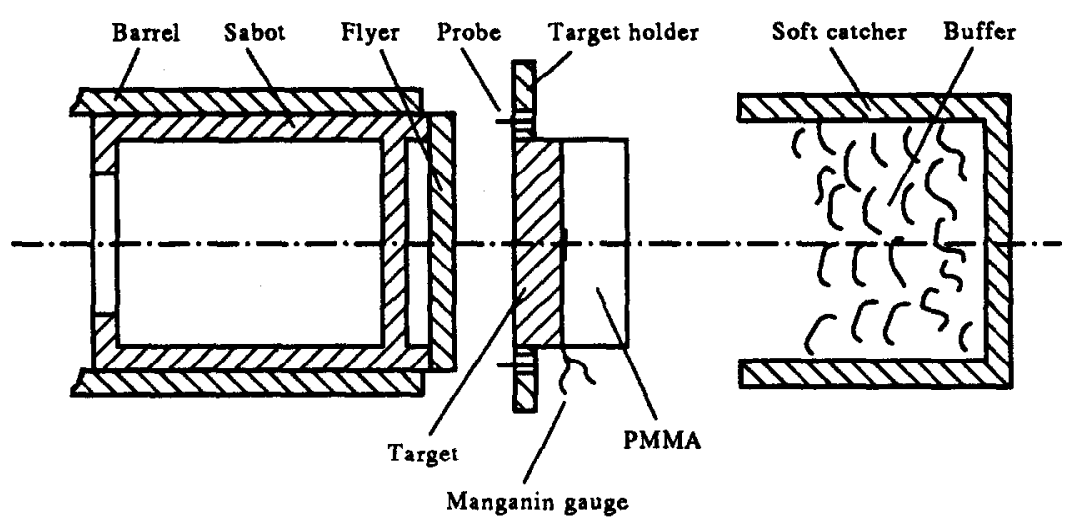

Fig. 4. Plate impact apparatus for spall study.

\subsection{One-dimensional finite-difference calculations}

The one-dimensional flow equations in terms of the Lagrangian position coordinate $x$ are

$$
\left\{\begin{array}{l}
\frac{\partial \varepsilon}{\partial t}+\frac{\partial u}{\partial x}=0 \\
\rho_{0} \frac{\partial u}{\partial t}-\frac{\partial \sigma}{\partial x}=0 \\
\rho_{0} \frac{\partial E}{\partial t}-\sigma \frac{\partial u}{\partial x}=0
\end{array}\right.
$$

where $\varepsilon \equiv 1-\left(\rho_{0} / \rho\right), t$ is the time, $\rho$ is the macroscopic average density of the porous materials ( $\rho_{0}$ is the initial density), $u$ is the particle velocity in the $x$ direction, $\sigma$ is the longitudinal stress component, and $E$ is the internal encrgy per unit mass.

The material constitutive equation is written in terms of the macroscopic mean stress $-P$ and the deviatoric stress components $S_{i j}$. In the model described here, void growth is related only to the mean stress $-P$ (pressure $P$ is assumed negative in tension). The deviatoric stress components depend on the shear modulus $\mu$ and yield strength $Y$, which are each functions of distention $\alpha$. The plastic yield condition for the porous materials is

$$
\frac{3}{2} S_{i j} S_{i j} \leqslant Y^{2}
$$

No attempt was made to include work-hardening and rate-dependent terms of the type used in eqn (12) for calculations of the wave profiles. These effects are still poorly understood themselves and do not greatly influence the fracture process. This treatment can greatly simplify calculation.

In the elastic region $\left(3 S_{i j} S_{i j}<2 Y^{2}\right)$ the stress deviatoric rates are given by

$$
\frac{\mathrm{d} S_{i j}}{\mathrm{~d} t}=2 \mu\left(\frac{\mathrm{d} \varepsilon_{i j}}{\mathrm{~d} t}-\frac{1}{3} \delta_{i j} \frac{\mathrm{d} \varepsilon}{\mathrm{d} t}\right)
$$

where $\varepsilon_{i j}$ is the macroscopic strain tensor component.

It is assumed that the function that relates pressure specific volume and specific internal energy for the matrix material in the porous state is the same as that which relates these quantities for the matrix material in its nonporous state (Carroll and Holt, 1972; Seaman et al., 1976). With this assumption, the $P-\alpha$ model gives the pressure in the porous material as a function of specific volume, specific internal energy, and porosity. The form of this 
(a)

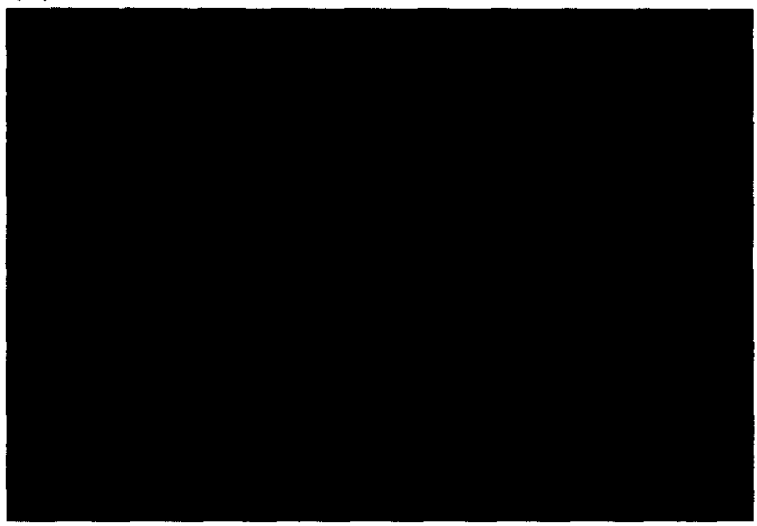

No.92-11 Vi $403.7 \mathrm{~m} / \mathrm{s}$

(b) Peak stress $1.18 \mathrm{GPa}$

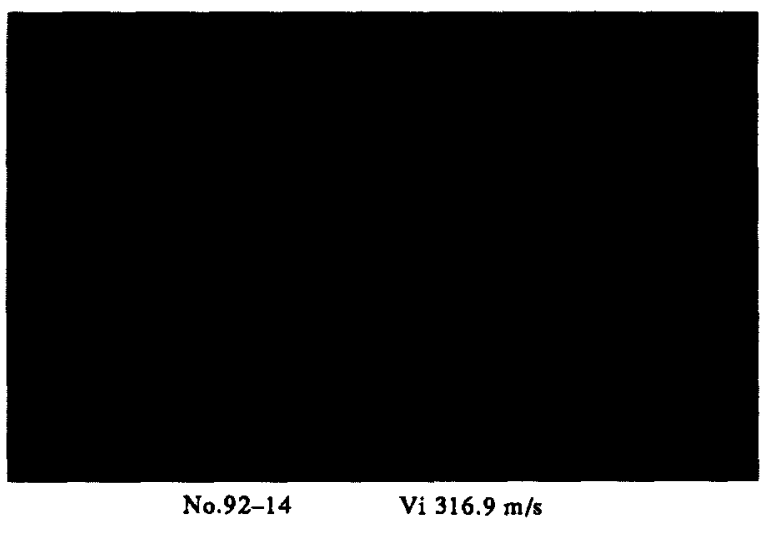

Peak stress 0.95 GPa

Fig. 5. Stress record from manganin gauge in PMMA behind aluminum alloy specimens. (a) Test 1 and (b) test 2 

Table 1

\begin{tabular}{|c|c|c|c|c|c|}
\hline $\begin{array}{l}\text { Test } \\
\text { no. }\end{array}$ & $\begin{array}{c}\text { Impact } \\
\text { velocity } \\
\left(\mathrm{mm} \mu \mathrm{s}^{-1}\right)\end{array}$ & Flyer & $\begin{array}{c}\text { Thickness } \\
\text { (mm) } \\
\text { Target }\end{array}$ & PMMA & $\begin{array}{c}\text { Peak stress } \\
\sigma_{\max } \\
(\text { GPa) }\end{array}$ \\
\hline 1 & 0.404 & 6.0 & 9.9 & 11.7 & 1.18 \\
\hline 2 & 0.317 & 6.3 & 9.8 & 11.9 & 0.95 \\
\hline
\end{tabular}

Table 2. Material parameters for spallation calculations

\begin{tabular}{ccc}
\hline & Aluminum & PMMA $^{*}$ \\
\hline$\rho_{0}\left(\mathrm{~g} \mathrm{~cm}^{-3}\right)$ & 2.78 & 1.185 \\
$K_{0}(\mathrm{GPa})$ & 79.57 & 8.13 \\
$\Gamma_{0}$ & 1.7 & \\
$Y_{0}(\mathrm{GPa})$ & 0.55 & \\
$H(\mathrm{GPa})$ & 0.5 & \\
$\mu_{\mathrm{s}}(\mathrm{GPa})$ & 25.0 & \\
$\gamma(\mathrm{GPa} \mu \mathrm{s})$ & 0.001 & \\
$\left.\gamma(\mathrm{GPa} \mathrm{cm})^{-2}\right)$ & $1 \times 10^{-4}$ & \\
$\alpha_{\text {crit }}$ & 1.35 & \\
\hline
\end{tabular}

* PMMA is treated in these calculations as a fluid (no shear strength).

function is determined by the pressure-specific volume-specific internal energy (PVE) function for the matrix material in its nonporous state. We use the following PVE relation for the porous material (Seaman et al., 1976) :

$$
P=P_{\mathrm{s}}(V / \alpha, E) / \alpha,
$$

where $V$ is the macroscopic specific volume for the porous material which is defined as $V=\rho_{0} / \rho$. The specific internal energy for the matrix material is the same in the porous and nonporous conditions, and the specific internal energy of the porous material is that of the matrix material, that is, the surface energy of the pores is neglected. The PVE relation of the matrix material is given by (Seaman et al., 1976)

$$
P_{\mathrm{s}}(V / \alpha, E)=K_{0}\left(\frac{\alpha}{V}-1\right)+\Gamma \rho_{\mathrm{s}} E
$$

where $K_{0}$ is the adiabatic bulk modulus at zero pressure, $\Gamma$ is the Gruneisen coefficient, and here $\rho_{s} \Gamma$ is assumed to be a constant given by its low-pressure value $\rho_{0} \Gamma_{0}$. Relation between the macroscopic yield strength $Y$ and distention $\alpha$ is given by (Johnson, 1981)

$$
Y=Y_{0} / \alpha
$$

The shear modulus is assumed to be degraded by the presence of voids. Relation between the shear modulus $\mu$ and distention $\alpha$ is suggested by (Mackenzie, 1950)

$$
\mu=\frac{\mu_{\mathrm{s}}}{\alpha}\left(1-\frac{6 K_{0}+12 \mu_{\mathrm{s}}}{9 K_{0}+8 \mu_{\mathrm{s}}} \frac{\alpha-1}{\alpha}\right)
$$

where $\mu_{\mathrm{s}}$ is the material shear modulus in the solid surrounding the void.

It is assumed that coalescence takes place when the distention $\alpha$ is equal to or greater than a threshold value $\alpha_{\text {crit }}$ which is given in Table 2 . We also assume that, when macroscopic stress $\Sigma$ [defined as eqn (28)] is equal or greater than the threshold stress $\Sigma_{\text {crit }}$ [eqn (31)], microvoids appear and begin to grow. Otherwise, the material is intact. 

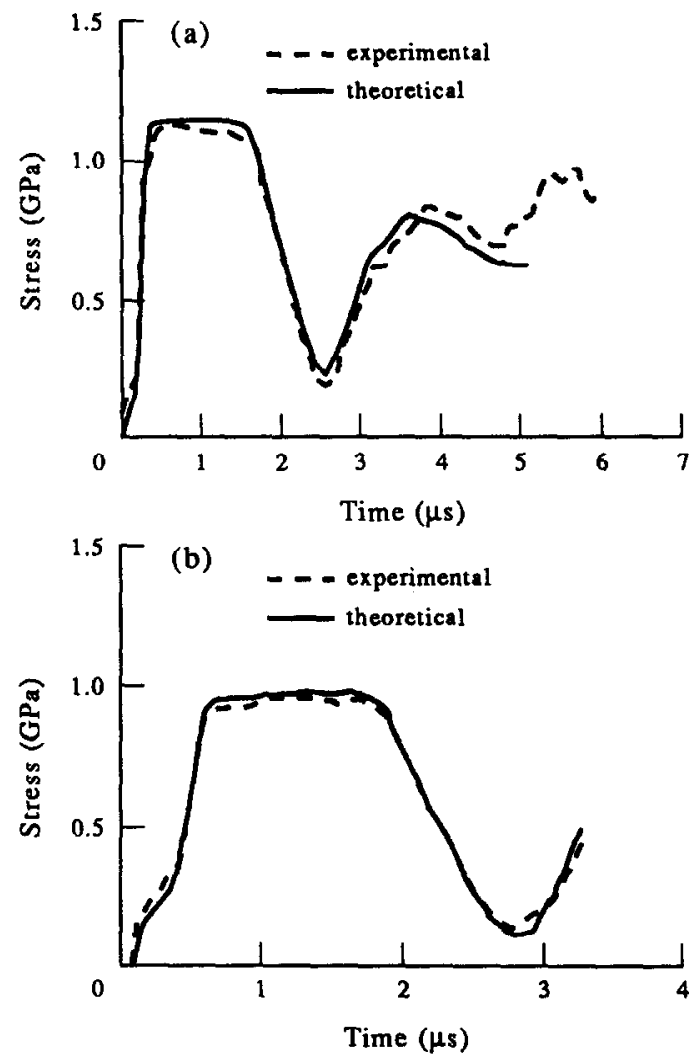

Fig. 6. Comparison of stress record from manganin gauge in PMMA behind aluminum alloy with computed stress. (a) Test 1 and (b) test 2 .

Application of the dynamic failure analysis to the problem of time-dependent spallation in aluminum alloy gives very good representations of the data (as shown in Fig. 6) with the material parameters listed in Table 2.

\section{DISCUSSION}

A great many experimental observations (Meyers and Aimone, 1983; Curran et al., 1987; Wang, 1993) show that the dynamic ductile fracture in solids is a complicated process which, in general, involves nucleation, growth and coalsecence of microvoids. The most common sites for void nucleation are hard second-phase particles or inclusions. The different distributions of hard second-phase particles or inclusions have a significant influence on the process of fracture in ductile materials. Besides these facts, rate-dependence of the materials, local inertial effects, thermal effects from the high rate plastic flow localization, as well as the physical properties of the materials are also very important factors in influencing the fracture modes. Description of the whole process of dynamic ductile fracture in detail is quite difficult or almost impossible. To develop a model to investigate the event of dynamic ductile fracture, we must make some assumptions. In our theoretical analysis, the following assumptions are made:

(1) The matrix is incompressible during pore growth. Based on the assumption of matrix incompressibility, we derived the pore-growth model. This assumption and the spherical geometry afforded a great simplification of the theoretical analysis. After comparing the porosity profiles, which were computed by a finite-difference computer code, for an incompressible hollow-sphere with those for a hollow aluminum sphere, Carroll and Holt (1972) suggested that compressibility of the matrix material greatly complicates the analysis, while the effect on the behavior of the porosity should not be great. Luk et al. (1991) used the models considering the material as incompressible and compressible to investigate dynamic spherical cavity expansion of strain-hardening materials. They found 
that, for an incompressible material, closed-form solutions could be obtained, whereas the compressible results required the numerical solution of differential equations. That is, it is difficult to obtain the closed form of the pore-growth relation if we consider the compressibility of the matcrials.

(2) The porous ductile material is statistically homogeneous and isotropic, that is, pores are fairly well distributed through the whole material. These assumptions neglect the distributions of microvoids with different radius. With these assumptions, we can study the behavior of dynamic damage in ductile materials by considering a hollow sphere of the matrix material of inner radius $a$ and outer radius $b$ or the ratio of total volume to matrix volume $\alpha$. Seaman et al. (1976) made detailed microscopic observations before and after shock-wave loading, and developed a statistical model of dynamic fracture called the NAG model. In the model, the distributions of radii of voids are included. But there are numerous phenomenological constants that are difficult to obtain. In fact, it is inconvenient to use the NAG model in practice.

(3) The equation of state for the material in the porous state is the same as in its nonporous state. Therefore, we can describe the behavior of dynamic damage and fracture in ductile materials in terms of continuum mechanics.

Thermal effects from high rate plastic deformation are not considered in our theoretical analysis. Johnson (1981) once numerically analyzed the change of temperature at expanding pore wall for copper-like material, and showed that if all the plastic work went into heat, the temperature at the expanding pore wall could be a substantial fraction of the melting temperature. Cortes (1992) investigated the thermal softening of the matrix material. He indicated that thermal softening was found to have a negligible influence on the dynamic tensile strength in the case of aluminum and copper-like materials, due to an excessively localized heat generation near the surface of the voids. How thermal effects influence the dynamic growth of a ductile void and how large thermal effects are comprise the work we want to carry on in the future.

The dynamic ductile model presented in this work contains many of the essential features of the process, such as rate-dependent sensitivity, inertial effects, etc. It is a simplified theoretical model in which only a few parameters ( $Y_{0}$ the yield strength, $\eta$ the viscosity coefficient, $H$ the linear work-hardening coefficient and $\gamma$ the density of void surface energy) need to be determined. This makes it easy to apply in studies of the behavior of dynamic ductile damage and fracture in solids.

\section{SUMMARY}

A dynamic ductile failure model is developed in which the inertial effects, the material rate sensitivity, the action of deviatoric stress and the effect of void surface energy are considered. Spall fracture of aluminum alloy for plate-impact conditions are successfully described with the model. Numerical calculations show that the inertial effects appear to resist the growth of voids. The higher the loading rates are, the greater the inertial effects are. It suggests that inertial effects should not be neglected in the whole process of spall fracture.

\section{REFERENCES}

Barber, T. M., Seaman. L., Crewdson, R. C. and Curran, D. R. (1972). Dynamic fracture criteria for ductile and brittle metals. J. Mater. 7, 393-401.

Carroll, M. M. and Holt, A. C. (1972). Static and dynamic pore-collapse relations for ductile porous materials. J. Appl. Phys. 43, 1626-1636.

Cochran, S. and Banner, D. (1977). Spall studies in uranium. J. Appl. Phys. 48, 2729-2737.

Cocks, A. C. F. (1989). Inelastic deformation of porous materials. J. Mech. Phys. Solids 37, 693-715.

Cortes, R. (1992). The ;rowth of microvoids under intense dynamic loading. Int. J. Solids Struct. 29, 13391349.

Curran, D. R., Seaman, L. and Shockey, D. A. (1977). Dynamic fracture in solids. Phys. Today 30, 46-55.

Curran, D. R., Seaman, L. and Shockey, D. A. (1987). Dynamic failure of solids. Phys. Rep. 147, 254-388.

Duva, J. M. and Hutchinson, J. W. (1984). Constitutive potentials for dilutely voided nonlinear materials. Mech. Mater. 3, 41-54.

Grady, D. E. (1988). The spall strength of condensed matter. J. Mech. Phys. Solids 36, 353-384. 
Gurson, A. L. (1977). Continuum theory of ductile rupture by void nucleation and growth. Part I-yield criteria and fiow rules for porous ductile media. J. Engng Mater. Technol. 99, 2-15.

Johnson, J. N. (1981). Dynamic fracture and spallation in ductile solids. J. Appl. Phys. 52, 2812-2825.

Johnson, W. and Mellor, P. B. (1973). Engineering Plasticity. Van Nostrand Reinhold, London.

Luk, V. K., Forrestal, M. J. and Amos, D. E. (1991). Dynamic spherical cavity expansion of strain-hardening materials. J. Appl. Mech. 58, 1-6.

Mackenzie, J. H. (1950). The elastic constants of a solid containing spherical holes. Proc. Phys. Soc. 63B, 2-11.

Meyers, M. A. and Aimone, C. T. (1983). Dynamic failure (spalling) of metals. Prog. Mater. Sci. 28, 1-96.

Nash, M. A. (1985). A model for ductile fracture in triaxial states of stress. RARDE Report $2 / 85$.

Nash, M. A. and Cullis, I. G. (1984). Numerical modelling of fracture-a model for ductile fracture in triaxial states of stress. 3rd Conf. Mech. Props. High Rates of Strain (Edited by J. Harding), pp. 307-314. Oxford.

Perzyna, P. (1986). Internal state variable description of dynamic fracture of ductile solids. Int. J. Solids Structures 22, 797-818.

Rajendran, A. M., Dietenberger, M. A. and Grove, D. J. (1989). A void growth-based failure model to describe spallation. J. Appl. Phys. 65, 1521-1527.

Rice, J. R. and Tracey, D. M. (1969). On the ductile enlargement of voids in triaxial stress fields. J. Mech. Phys. Solids 17, 201-217.

Seaman, L., Curran, D. R. and Shockey, D. A. (1976). Computational models for ductile and brittle fracture. $J$. Appl. Phys. 47, 4814-4826.

Tvergaard, V. and Needleman, A. (1984), Analysis of the cup-cone fracture in a round tensile bar. Acta Metall. 32, $157-169$.

Wang, Z.-P. (1993). A study on one-dimensional dynamic damage in pure copper. Acta Mech. Solida Sinica(English edn) 6, 69-80. 